\title{
Weighted Tardiness Minimization in Job Shops with Setup Times by Hybrid Genetic Algorithm
}

\author{
Miguel A. González, Camino R. Vela, and Ramiro Varela \\ Department of Computer Science, \\ University of Oviedo, (Spain) Campus de Viesques s/n, Gijón, 33271, Spain \\ http://www.aic.uniovi.es/tc
}

\begin{abstract}
In this paper we confront the weighted tardiness minimization in the job shop scheduling problem with sequence-dependent setup times. We start by extending an existing disjunctive graph model used for makespan minimization to represent the weighted tardiness problem. Using this representation, we adapt a local search neighborhood originally defined for makespan minimization. The proposed neighborhood structure is used in a genetic algorithm hybridized with a simple tabu search method. This algorithm is quite competitive with state-of-theart methods in solving problem instances from several datasets of both classical JSP and JSP with setup times.
\end{abstract}

\section{Introduction}

In this paper we confront the Job Shop Scheduling Problem with SequenceDependent Setup Times (SDST-JSP) with weighted tardiness minimization. JSP has interested to researchers over the last decades, but in most cases the objective function is the makespan, even though other objective functions such as weighted tardiness or total flow time are sometimes more important in many reallife problems. Also, setup considerations are a relevant characteristic of many real scheduling problems that add to the difficulty of solving these problems with respect to their non-setup counterparts. Incorporating sequence-dependent setup times changes the nature of scheduling problems, so well-known results and techniques for the JSP are not directly applicable to the SDST-JSP. Some extensions have been done for makespan minimization in 1613.

As far as we know, the best approach to weighted tardiness minimization in the SDST-JSP is the presented by Sun and Noble in [10. They propose a shifting bottleneck algorithm and in their experimental study this algorithm is compared with very simple heuristics (some priority rules) across randomly generated instances that are not available for further comparison. However, weighted tardiness has been largely considered for the classic JSP, maybe the algorithms proposed in [3] and [7] being the most relevant approaches currently. In [3], Essafi et al. propose a hybrid genetic algorithm that uses a local search based in reversing critical arcs and an algorithm that iterates between hill climbing and random generation of neighbors to escape from local optima. In [7] Mati et al. propose a local search method that uses estimators to evaluate the neighbors and 
that is capable of minimizing any regular objective function, i.e. a non-decreasing function on the completion time of the operations.

We propose a hybrid algorithm that combines a genetic algorithm (GA) with tabu search (TS). The core of this algorithm is a variation of the neighborhood structure $N_{1}^{S}$ introduced in 13 . for the SDST-JSP with makespan minimization, which in its turn extends the structures proposed in [12 for the classical JSP. We define a disjunctive graph model for the SDST-JSP with weighted tardiness minimization to formalize this neigborhood. The proposed algorithm is termed $G A+T S$ in the following. We also define a method for estimating the weighted tardiness of the neighbors, and we will see that this estimation is less accurate and more time consuming than similar estimations for the makespan, due to the difference in the problem difficulty. We have conducted an experimental study across conventional benchmarks to compare $G A+T S$ with state-of-theart algorithms in both classical JSP and SDST-JSP. The results show that the proposed algorithm outperforms the other methods.

The rest of the paper is organized as follows. In Section 2 we formulate the JSP and introduce the notation used across the paper. In section 3 we describe the main components of the genetic algorithm. The proposed neighborhood structure, the weighted tardiness estimation algorithm and the main components of the TS algorithm are described in Section 4 . Section 5 reports results from the experimental study. Finally, in Section [6] we summarize the main conclusions and propose some ideas for future work.

\section{Description of the Problem}

In the job shop scheduling problem, a set of $N$ jobs, $J=\left\{J_{1}, \ldots, J_{N}\right\}$, are to be processed on a set of $M$ machines (resources), $R=\left\{R_{1}, \ldots, R_{M}\right\}$ while minimizing some function of completion times of the jobs, subject to constraints: (i) the sequence of machines for each job is prescribed, and (ii) each machine can process at most one job at a time. The processing of a job on a machine is called an operation, and its duration is a given constant. We denote by $p_{u}$ the processing time of operation $u$. A time may be needed to adjust a machine between two consecutive operations, which is called a setup time, and which may or may not be sequence-dependent. We adopt the following notation for the setup times: $S_{u v}$ is the setup time between consecutive operations $u, v \in R_{j}$, and $S_{0 u}$ is the setup time required before $u$ if this operation is the first one scheduled on his machine. A job $J_{i}$ may also have a due date $d_{i}$, that is a time before jobs should be completed, and a weight $w_{i}$, that is the relevance of the job. The objective here is to minimize the weighted cost of the jobs exceeding its due-dates, also known as the weighted tardiness. In the following, we denote by $t_{u}$ the starting time of operation $u$, that needs to be determined.

The SDST-JSP has two binary constraints: precedence and capacity. Precedence constraints, defined by the sequential routings of the tasks within a job, translate into linear inequalities of the type: $t_{u}+p_{u} \leq t_{v}$, if $v$ is the next operation to $u$ in the job sequence. Capacity constraints that restrict the use of each 
resource to only one task at a time translate into disjunctive constraints of the form: $t_{u}+p_{u}+S_{u v} \leq t_{v} \vee t_{v}+p_{v}+S_{v u} \leq t_{u}$, where $u$ and $v$ are operations requiring the same machine.

The objective is to obtain a feasible schedule that minimizes the weighted tardiness, defined as:

$$
\sum_{i=1, \ldots, N} w_{i} T_{i}
$$

where $T_{i}$ is the tardiness of the job $i$, defined as $T_{i}=\max \left\{C_{i}-d_{i}, 0\right\}$, where $C_{i}$ is the completion time of job $i$. This problem is denoted by $J\left|s_{i j}\right| \sum w_{i} T_{i}$ in the literature.

\subsection{The Disjunctive Graph Model Representation}

The disjunctive graph is a common representation in scheduling, its exact definition depending on the particular problem. For the $J\left|s_{i j}\right| \sum w_{i} T_{i}$ problem, we propose that it be represented by a directed graph $G=\left(V, A \cup E \cup I_{1} \cup I_{2}\right)$. Each node in set $V$ represents a task of the problem, with the exception of the dummy nodes start and end $1 \leq i \leq N$, which represent fictitious operations that do not require any machine. Arcs in $A$ are called conjunctive arcs and represent precedence constraints while arcs in $E$ are called disjunctive arcs and represent capacity constraints. Set $E$ is partitioned into subsets $E_{i}$, with $E=\cup_{j=1, \ldots, M} E_{j}$, where $E_{j}$ corresponds to resource $R_{j}$ and includes two directed $\operatorname{arcs}(v, w)$ and $(w, v)$ for each pair $v, w$ of operations requiring that resource. Each $\operatorname{arc}(v, w)$ in $A$ is weighted with the processing time of the operation at the source node, $p_{v}$, and each $\operatorname{arc}(v, w)$ of $E$ is weighted with $p_{v}+S_{v w}$. Set $I_{1}$ includes arcs of the form $\left(\right.$ start, $v$ ) for each operation $v$ of the problem, weighted with $S_{0 v}$. Set $I_{2}$ includes $\operatorname{arcs}\left(\omega(i)\right.$, end $\left._{i}\right), 1 \leq i \leq N$, weighted with $p_{\omega(i)}$, where $\omega(i)$ is the last operation of job $J_{i}$

A feasible schedule is represented by an acyclic subgraph $G_{s}$ of $G, G_{s}=$ ( $V, A \cup H \cup J_{1} \cup I_{2}$ ), where $H=\cup_{j=1 \ldots M} H_{j}, H_{j}$ being a minimal subset of arcs of $E_{j}$ defining a processing order for all operations requiring $R_{j}$ and where $J_{1}$ consists of arcs $\left(\right.$ start,$\left.v_{j}\right), j=1 \ldots M, v_{j}$ being the first operation of $H_{j}$. Finding a solution can thus be reduced to discovering compatible orderings $H_{j}$, or partial schedules, that translate into a solution graph $G_{s}$ without cycles. Figure 1 shows a solution to a problem with 3 jobs and 3 machines; dotted arcs belong to $H$ and $J_{1}$, while continuous arcs belong to $A$.

To calculate the weighted tardiness of the schedule we have to compute the cost of a critical path in $G_{s}$ to each node $e n d_{i} 1 \leq i \leq N$, i.e., a directed path in $G_{s}$ from node start to node $e d_{i}$ having maximum cost. Nodes and arcs in a critical path are also termed critical. A critical path may be represented as a sequence of the form start, $B_{1}, \ldots, B_{r}$, end $d_{i} 1 \leq i \leq N$, where each $B_{k}$, $1 \leq k \leq r$, is a critical block, a maximal subsequence of consecutive operations in the critical path requiring the same machine. 


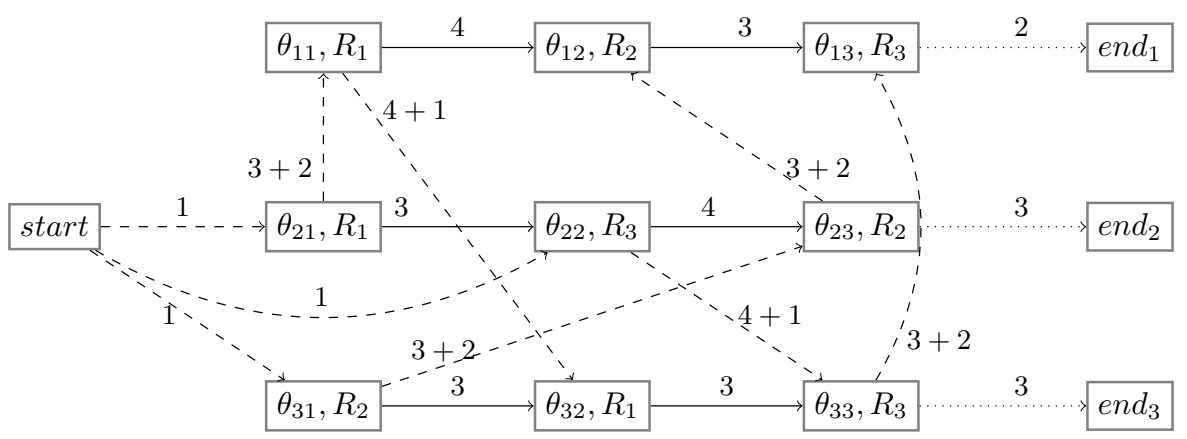

Fig. 1. A feasible schedule to a problem with 3 jobs and 3 machines

In order to simplify expressions, we define the following notation for a feasible schedule. Given a solution graph $G_{s}$ for the SDST-JSP, the head of an operation $v$, denoted $r_{v}$, is the cost of the longest path from node start to node $v$, i.e., the starting time of $v$ in the schedule represented by $G_{s}$. A tail $q_{v}^{i}, 1 \leq i \leq N$ is the cost of the longest path from node $v$ to node $e n d_{i}$, minus the duration of task in node $v$. For practical reasons we will take $q_{v}^{i}=-\infty$ when no path exist from $v$ to end $_{i}$. Here, it is important to remark that we have had to define $N$ tails for each operation, while for makespan minimization it is required just one. Let $P J_{v}$ and $S J_{v}$ denote respectively the predecessor and successor of $v$ in the job sequence, and $P M_{v}$ and $S M_{v}$ the predecessor and successor of $v$ in its machine sequence. We take node start to be $P J_{v}$ for the first task of every job and $P M_{v}$ for the first task to be processed in each machine; note that $p_{\text {start }}=0$. Then, the head of every operation $v$ and every dummy node may be computed as follows:

$$
\begin{gathered}
r_{\text {start }}=0 \\
r_{v}=\max \left(r_{P J_{v}}+p_{P J_{v}}, r_{P M_{v}}+p_{P M_{v}}+S_{P M_{v} v}\right) \\
r_{e n d_{i}}=r_{v}+p_{v},\left(v, \text { end }_{i}\right) \in I_{2}, 1 \leq i \leq N
\end{gathered}
$$

Similarly, for $1 \leq i \leq N$, we take node $e n d_{i}$ as $S J_{v}$ for the last task of job $i$, and $p_{\text {end }}=0$. So, the tail of all operations are computed as follows:

$$
\begin{aligned}
& q_{\text {endi }}^{i}=0 \\
& q_{\text {endi }}^{j}=-\infty, j \neq i
\end{aligned}
$$

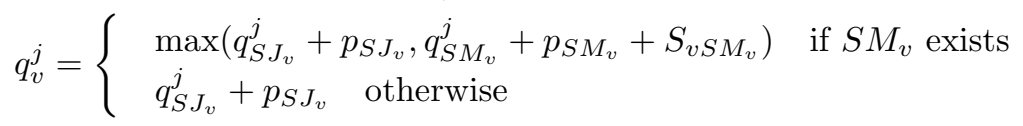

$$
\begin{aligned}
& q_{\text {start }}^{j}=\max _{v \in S M_{\text {start }}}\left\{q_{v}^{j}+p_{v}+S_{0 v}\right\}
\end{aligned}
$$




\section{Genetic Algorithm for the SDST-JSP}

In this paper we will use a conventional GA, with permutations with repetition as our encoding schema and Job Order Crossover (JOX) for chromosome mating, uniform selection and generational replacement using tournament between two parents and two offspring. To build schedules we have used the Serial Schedule Generation Schema (SSGS) proposed in 1] for the SDST-JSP. SSGS iterates over the operations in the chromosome sequence and assigns each one the earliest starting time that satisfies all constraints with respect to previously scheduled operations. SSGS produces active schedules, provided that the triangular inequality for the setup times holds for all operations requiring the same machine [1, and this is the case in the instances used in our experimental study.

When combined with GA, TS is applied to every schedule produced by SSGS. Then, the chromosome is rebuilt from the improved schedule obtained by TS, so as its characteristics can be transferred to subsequent offspring. This effect of the evaluation function is known as Lamarckian evolution.

\section{Tabu Search for the Weighted Tardiness Minimization in the SDST-JSP}

We use here a conventional TS algorithm [4, the particular implementation is borrowed from [5]. In the next subsections we describe in detail the components of the algorithm that has been adapted to the SDST-JSP with weighted tardiness minimization, namely the neighborhood structure and the procedure for weighted tardiness estimation after a move.

\subsection{The Neighborhood Structure}

As usual, this structure is based on changing processing orders in a critical block. However, the number of critical paths in a problem instance is usually large and so not all candidate moves can be considered in order to obtain a reasonable number of neighbors. To do that, we consider two options: all critical paths corresponding to tardy jobs or just the critical path that most contributes to the objective function. We have observed that neither option is clearly better than the other in some preliminary experiments. Moreover, it happens that there are substantial differences that depend on the instances, so we have finally opted to consider them both and choose randomly each time the TS algorithm is issued.

Also, we have opted to consider a neighborhood structure that generates a small number of neighbors from each critical block. For this reason, we adapted the structure $N_{1}^{S}$ proposed in [13] for SDST-JSP with makespan minimization, which is based on previous structures given in [8] and [12 for the standard JSP. This structure can be formalized for the SDST-JSP with weighted tardiness minimization from the disjunctive model defined in 2.1] $N_{1}^{S}$ is based on the following results. 
Proposition 1. Let $H$ be a schedule and $(v, w)$ a disjunctive arc that is not in a critical block. Then, reversing the arc $(v, w)$ does not produce any improvement, provided that the triangular inequality for the setup times holds for all operations requiring the same machine.

So we have to reverse a critical arc to obtain an improving schedule. In [13] the authors define non-improving conditions for some reversals of critical arcs in makespan optimization that in principle can not be translated to the weighted tardiness case. Regarding feasibility, the next result gives a sufficient condition for an alternative path not existing after the reversal of a critical arc. If such an alternative path exists then the resulting neighbor would be unfeasible because it would contain a cycle.

Proposition 2. Let $H$ be a schedule and $(v, w)$ an arc in a critical block. A sufficient condition for an alternative path between $v$ and $w$ not existing is that

$$
r_{P J_{w}}<r_{S J_{v}}+p_{S J_{v}}+\min \left\{S_{k l} \mid(k, l) \in E, J_{k}=J_{v}\right\}
$$

where $J_{k}$ is the job of operation $k$.

So, the neighborhood structure $N_{1}^{S}$ is defined as follows.

Definition 1. $\left(N_{1}^{S}\right)$ Given a schedule $H$, the neighborhood $N_{1}^{S}(H)$ consists of all schedules derived from $H$ by reversing one arc $(v, w)$ of a critical block, provided that feasibility condition given in proposition 2 holds.

\subsection{Weighted Tardiness Estimation}

Even though computing the weighted tardiness of a neighbor only requires to recompute heads (tails) of operations that are after (before) the first (last) operation moved, for the sake of efficiency the selection rule is based on weighted tardiness estimations instead of computing the actual weighted tardiness of all neighbors. For this purpose, we have extended the procedure lpath given for the JSP in [1] to cope with both setup times and weighted tardiness. This procedure is termed lpathSWT and it is shown in Algorithm 1 .

Remember that each task $t$ has $N$ tails denoted by $q_{t}^{1} \ldots q_{t}^{N}$. For each $i=$ $1 \ldots N$, lpath $S W T$ estimates the cost of the longest path from node start to each node $e n d_{i}$ through the node $v$ or the node $w$, and then estimates the weighted tardiness of the neighboring schedule from the estimations of these paths. It's easy to prove that lpathSWT produces a lower bound for the weighted tardiness when using $N_{1}^{S}$.

The makespan estimation algorithm lpath is very accurate and very efficient. However, lpathSWT is much more time consuming as it calculates $N$ tails for each operation. Moreover, experiments conclude that weighted tardiness estimation is much less accurate than makespan estimation. We have conducted a series of experiments in several instances, generating 3 millions of neighbors for each instance, and for $81.56 \%$ of neighbors the makespan estimation coincided 


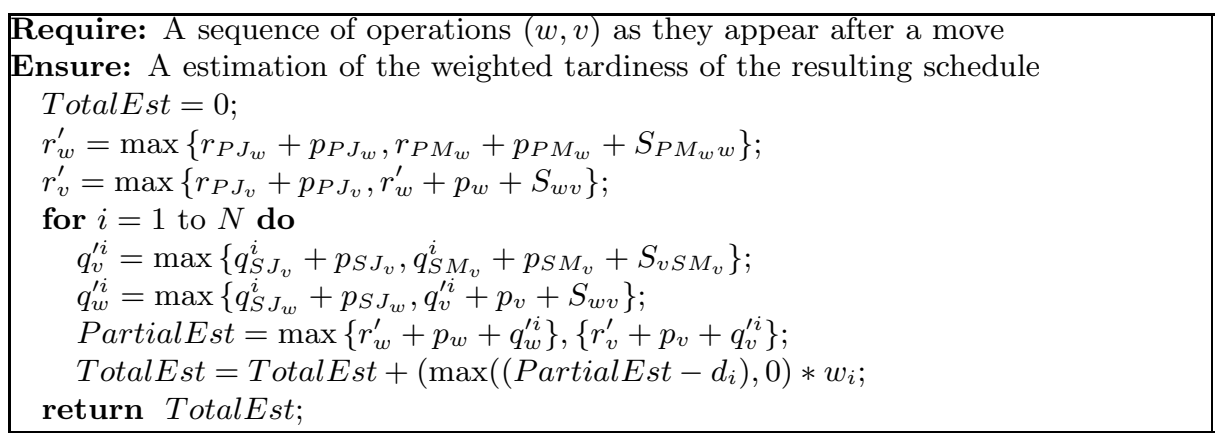

Alg. 1. Procedure lpathSWT

with the actual value, but for weighted tardiness this value drops to $51.37 \%$ of the cases.

Other authors, for example Mati et al. in 7] or Essafi et al. in 3] opted to use more accurate estimations (they report results with exact estimations from $57 \%$ to $76 \%$, depending on the particular instance). However, their estimation procedure is more time consuming as the complexity goes up from $O(1)$ to $O(N)$ for each path, where $N$ is the number of jobs.

For these reasons, we have opted to evaluate the actual weighted tardiness when the neighbor's estimation is lower than the actual weighted tardiness of the original schedule. So, the use of lpathSWT allows the algorithm to discard a lot of neighbors in a very fast manner. Some preliminary results have shown that the improvement achieved in this way makes up the time consumed by far.

\section{Experimental Study}

The purpose of the experimental study is to compare $G A+T S$ with other stateof-the-art algorithms. Firstly, we compare our algorithm with the $G L S$ algorithm proposed in [3] and the $M D L$ algorithm proposed in [7] to solve the JSP. We experimented across the 22 instances of size $10 \times 10$ proposed by Singer and Pinedo in 9] (ABZ5, ABZ6, LA16 to LA24, MT10, and ORB01 to ORB10). Weights and due dates are defined as follows: the first $20 \%$ of the jobs have a weight 4 (very important jobs), the next $60 \%$ have weight 2 (moderately important jobs) and the remaining jobs have weight 1 (not important jobs). The due date $d_{i}$ for each job $i$ is defined in this way:

$$
d_{i}=f * \sum_{j=1}^{M} p_{i j},
$$

where $f$ is a parameter that controls the tightness of the due dates. In this benchmark three values are considered: $f=1.3, f=1.5$ and $f=1.6$.

The algorithm proposed by Essafi et al. is implemented in $\mathrm{C}++$ and the experiments are carried out in a $\mathrm{PC}$ with a $2.8 \mathrm{GHz}$ processor and $512 \mathrm{MB}$ RAM, 
giving a maximum runtime of 18 seconds per run. The local search proposed by Mati et al. runs in a Pentium with a $2.6 \mathrm{GHz}$ processor, and they use a maximum runtime of 18 seconds too. $G A+T S$ runs in a Windows XP in a Intel Core 2 Duo at $2.66 \mathrm{GHz}$ with $2 \mathrm{~Gb} \mathrm{RAM}$. We choose the parameters /58/70/50/ (/GA population/GA generations/maximum number of iterations without improvement for TS/) for $G A+T S$ to obtain a similar runtime.

Table 1 summarizes the results of these experiments; 10 trials were done for each instance and the average weighted tardiness of the 10 solutions and the number of times that the best known solution (BKS) was found are reported, "." indicates that BKS is reached in all 10 trials.

Table 1. Results from $G L S, M D L$ and $G A+T S$ across Singer and Pinedo's instances

\begin{tabular}{|c|c|c|c|c|c|c|c|c|c|c|c|c|}
\hline \multirow[b]{2}{*}{ Inst. } & \multirow[b]{2}{*}{ BKS } & \multicolumn{3}{|c|}{$=1.3$} & \multicolumn{4}{|c|}{$\overline{f=1.5}$} & \multicolumn{4}{|c|}{$=1.6$} \\
\hline & & GLS & MDL & $\mathrm{GA}+\mathrm{TS}$ & BKS & GLS & MDL & GA- & BKS & GLS & MDL & $\mathrm{GA}+\mathrm{TS}$ \\
\hline$\overline{\mathrm{ABZ5}}$ & 1403 & $=$ & $\overline{1414(2)}$ & $\overline{1412(7)}$ & $\overline{\overline{69}}$ & - & - & & $\overline{0}$ & $=$ & - & \\
\hline $3 Z 6$ & & - & - & 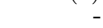 & . & & . & & 0 & 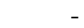 & - & - \\
\hline & 1169 & $175(9)$ & - & - & 166 & & - & & 0 & 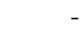 & - & - \\
\hline 17 & 899 & - & - & - & 260 & & - & & 65 & - & - & - \\
\hline 18 & 9 & $933(6)$ & $934(6)$ & & 34 & & . & - & 0 & - & - & - \\
\hline LA19 & 948 & $949(8)$ & - & $998(4)$ & 21 & & - & & 0 & - & - & - \\
\hline & 805 & - & - & $834(3)$ & 0 & & - & $0(7)$ & 0 & - & - & - \\
\hline 1 & 463 & - & - & - & 0 & & - & 7 & U & - & - & - \\
\hline 22 & 1064 & 1087(1) & $1077(4)$ & $1079(3)$ & 196 & & - & & 0 & 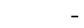 & - & - \\
\hline LA 23 & 835 & $865(2)$ & $865(2)$ & $870(1)$ & 2 & & - & & 0 & - & - & - \\
\hline 24 & 835 & & - & & 82 & $86(3)$ & $86(2)$ & $88(1)$ & 0 & - & - & - \\
\hline 10 & 1363 & $1372(9)$ & - & 1383(9) & 394 & - & & 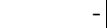 & 141 & $162(1)$ & 152(1) & $145(6)$ \\
\hline & 68 & $2651(0)$ & $2639(3)$ & $2578(8)$ & 1098 & $1159(6)$ & $1247(0)$ & - & 566 & $688(0)$ & $653(0)$ & (2) \\
\hline Q & 8 & $1444(2)$ & $1426(3)$ & $1426(3)$ & 292 & - & - & & 44 & - & - & \\
\hline 33 & 2111 & $2170(4)$ & 2158(1) & (6) & 918 & $943(4)$ & $961(0)$ & 939(4) & 422 & $514(1)$ & $463(4)$ & $434(7)$ \\
\hline 34 & 23 & $1643(\mathbf{7})$ & $1690(2)$ & $1632(6)$ & 358 & $394(8)$ & $435(4)$ & & 66 & $78(8)$ & $68(8)$ & \\
\hline ORB5 & 1593 & 1659(1) & $1775(0)$ & $1615(7)$ & 405 & - & $415(8)$ & (7) & 163 & $181(0)$ & $176(3)$ & $176(3)$ \\
\hline ORB6 & 1790 & & $1793(9)$ & $1854(5)$ & 426 & $440(5)$ & $437(5)$ & $430(8)$ & 28 & - & - & \\
\hline & 590 & $2(9)$ & - & & 50 & $55(8)$ & - & & 0 & - & - & \\
\hline ORB8 & 2429 & $2522(0)$ & $2523(0)$ & $2477(4)$ & 1023 & $1059(7)$ & $1036(6)$ & (2) & 621 & $669(0)$ & $643(1)$ & $639(3)$ \\
\hline & 1316 & & & & 297 & $311(7)$ & $299(9)$ & & 66 & $83(7)$ & $80(4)$ & \\
\hline ORB10 & 1679 & 1718(5) & 1774(1) & $1731(\mathbf{6})$ & 346 & $400(4)$ & $436(0)$ & $430(2)$ & 76 & $142(0)$ & $117(0)$ & $82(5)$ \\
\hline
\end{tabular}

$G A+T S$ was the only algorithm capable of reaching the BKS in at least one run for all 66 instances. Globally, $G A+T S$ obtains the BKS in 517 of the total 660 runs (78.3\%), GLS in $443(67.1 \%)$ and MDL in 458 (69.4\%). Regarding the average weighted tardiness, we have run two t-tests with alpha level at 0.05 to compare $G A+T S$ against GLS and MDL. With p-values of 0.016 and 0.010 respectively, both tests showed that there is good evidence that the mean average weighted tardiness obtained by $G A+T S$ is lower than the obtained by the other methods. Overall, $G A+T S$ is quite competitive with the state-of-the-art algorithms in solving the classic JSP with weighted tardiness minimization.

In the second series of experiments, we compared $G A+T S$ with ILOG CPLEX CP Optimizer (CP) in solving the SDST-JSP across the BT-set proposed in [2]. We define due dates and weights as before. BT instances are divided in three groups depending on its size: small instances, t2-ps01 to t2-ps05, are $10 \times 5$, medium instances, t2-ps06 to t2-ps10, are $15 \times 5$, and large instances, t2-ps11 to $\mathrm{t} 2$-ps 15 , are $20 \times 5$. These instances verify the triangular inequality for setup times. $G A+T S$ was parameterized as $/ 100 / 200 / 50 /$. CP was run setting the 
option "Extended" for parameter "NoOverlapInferenceLevel" as the results in this case were slightly better. Both methods were run 30 times for each instance. Table (2) summarizes the results of these experiments: for each method and instance, the average value of the weighted tardiness is reported. The time taken by $G A+T S$ in a single run is given in the last column. CP was given this time plus $20 \%$ more in each run. As we can observe, $G A+T S$ reaches much better solutions than $C P$. On average, the weighted tardiness obtained by $C P$ is $13.6 \%$ worse than that obtained by $G A+T S$. We have run a t-test with alpha level at 0.05 , and with a p-value of 0.000 the test showed that there is strong evidence that the mean average weighted tardiness obtained by $G A+T S$ is lower than the obtained by CP.

Table 2. Results from $G A+T S$ and $C P$ in solving SDST-JSP with weighted tardiness minimization on the BT-set

\begin{tabular}{l|rr|rr|rr|r}
\hline \hline & \multicolumn{2}{|c}{$f=1.3$} & \multicolumn{2}{c}{$f=1.5$} & \multicolumn{3}{c}{$f=1.6$} \\
Inst. & GA+TS & CP & GA+TS & CP & GA+TS & CP & T(s) \\
\hline \hline t2-ps01 & 4454 & 4994 & 3361 & 3911 & 2852 & 3506 & 47 \\
t2-ps02 & 3432 & 4143 & 2674 & 2957 & 2301 & 2558 & 48 \\
t2-ps03 & 4001 & 4609 & 3120 & 3560 & 2677 & 3143 & 51 \\
t2-ps04 & 3732 & 4021 & 2890 & 3050 & 2539 & 2632 & 48 \\
t2-ps05 & 3806 & 4445 & 2996 & 3532 & 2620 & 3038 & 45 \\
t2-ps06 & 9941 & 10533 & 8238 & 8997 & 7436 & 8148 & 121 \\
t2-ps07 & 9508 & 10552 & 8079 & 8875 & 7425 & 8642 & 122 \\
t2-ps08 & 9902 & 10834 & 8360 & 9317 & 7624 & 8521 & 120 \\
t2-ps09 & 9998 & 11569 & 8215 & 9697 & 7317 & 8813 & 124 \\
t2-ps10 & 10569 & 11999 & 8745 & 9968 & 7914 & 8848 & 116 \\
t2-ps11 & 23052 & 26169 & 20816 & 24132 & 19764 & 22909 & 229 \\
t2-ps12 & 23158 & 25331 & 21119 & 22309 & 20106 & 21039 & 241 \\
t2-ps13 & 24026 & 25729 & 21821 & 23548 & 20618 & 22279 & 244 \\
t2-ps14 & 25416 & 27569 & 23051 & 25580 & 21892 & 24079 & 250 \\
t2-ps15 & 25427 & 27144 & 23028 & 25450 & 22049 & 23919 & 237 \\
\hline \hline
\end{tabular}

\section{Conclusions}

Our study of SDST-JSP has demonstrated that metaheuristics such as genetic algorithms and tabu search are very efficient in solving complex scheduling problems. These techniques are flexible and robust, so as they can be adapted to the particular characteristics of a given problem. Here, we have seen how a solution designed to cope with makespan can be adapted to cope with weighted tardiness, which is well-known that is harder to optimize. Also, we have demonstrated that for weighted tardiness minimization, a specific solution based on specific knowledge of the problem domain can be much more efficient than a solution built on a general purpose solver.

As future work, we plan to consider other scheduling problems and different objective functions, even non-regular objective functions, i.e. objective functions that could be decreasing on the completion times of the operations, such as robustness or stability measures. 
Acknowledgments. This research has been supported by the Spanish Ministry of Science and Innovation under research project MICINN-FEDER TIN201020976-C02-02 and by the Principality of Asturias under grant FICYT-BP07-109.

\section{References}

1. Artigues, C., Lopez, P., Ayache, P.D.: Schedule generation schemes for the job shop problem with sequence-dependent setup times: Dominance properties and computational analysis. Annals of Operations Research 138, 21-52 (2005)

2. Brucker, P., Thiele, O.: A branch and bound method for the general-job shop problem with sequence-dependent setup times. Operations Research Spektrum 18, 145-161 (1996)

3. Essafi, I., Mati, Y., Dauzère-Pérès, S.: A genetic local search algorithm for minimizing total weighted tardiness in the job-shop scheduling problem. Computers and Operations Research 35, 2599-2616 (2008)

4. Glover, F., Laguna, M.: Tabu Search. Kluwer Academic Publishers (1997)

5. González, M.A., Vela, C.R., Varela, R.: Genetic Algorithm Combined with Tabu Search for the Job Shop Scheduling Problem with Setup Times. In: Mira, J., Ferrández, J.M., Álvarez, J.R., de la Paz, F., Toledo, F.J. (eds.) IWINAC 2009. LNCS, vol. 5601, pp. 265-274. Springer, Heidelberg (2009)

6. González, M.A., Vela, C., Varela, R.: A new hybrid genetic algorithm for the job shop scheduling problem with setup times. In: Proceedings of the Eighteenth International Conference on Automated Planning and Scheduling (ICAPS 2008), pp. 116-123. AAAI Press, Sidney (2008)

7. Mati, Y., Dauzere-Peres, S., Lahlou, C.: A general approach for optimizing regular criteria in the job-shop scheduling problem. European Journal of Operational Research (2011), doi:10.1016/j.ejor.2011.01.046

8. Matsuo, H., Suh, C., Sullivan, R.: A controlled search simulated annealing method for the general jobshop scheduling problem. Working paper 03-44-88, Graduate School of Business, University of Texas (1988)

9. Singer, M., Pinedo, M.: A computational study of branch and bound techniques for minimizing the total weighted tardiness in job shops. IIE Transactions 30, 109-118 (1998)

10. Sun, X., Noble, J.: An approach to job shop scheduling with sequence-dependent setups. Journal of Manufacturing Systems 18(6), 416-430 (1999)

11. Taillard, E.: Parallel taboo search techniques for the job shop scheduling problem. ORSA Journal on Computing 6, 108-117 (1993)

12. Van Laarhoven, P., Aarts, E., Lenstra, K.: Job shop scheduling by simulated annealing. Operations Research 40, 113-125 (1992)

13. Vela, C.R., Varela, R., González, M.A.: Local search and genetic algorithm for the job shop scheduling problem with sequence dependent setup times. Journal of Heuristics 16, 139-165 (2010) 